\title{
Some results on non-commutative Banach function spaces II (Infinite cases)
}

Dedicated to Professor Tsuyoshi Ando on his sixtieth birthday

\author{
Keiichi WATANABE* \\ (Received August 7, 1992)
}

\begin{abstract}
An extension of Fatou's lemma for generalized $s$-numbers of measurable operators associated with a semifinite von Neumann algebra is obtained. We extend several fundamental results on non-commutative Banach function spaces associated with finite von Neumann algebras to those of semifinite cases. They are such as correspondence between rearrangement invariant function norms and non-commutative Banach function spaces, heredity of absolute continuity of norms and separability.
\end{abstract}

\section{Introduction}

Let $\mathscr{M}$ be a semifinite von Neumann algebra with a faithful normal semifinite trace $\tau$.

We defined in [W2] a system of axioms for non-commutative Banach function spaces, and we proved that if $\mathscr{M}$ is a $\mathrm{II}_{1}$-factor then non-commutative Banach function spaces bijectively correspond to rearrangement invariant function norms on $(0, \tau(1))$. We also obtained a necessary and sufficient condition for a non-commutative Banach function space to be separable.

Our aim in this article is to extend the results obtained in [W2] to general $\sigma$-finite semifinite von Neumann algebras by a simple device related to convergence in measure.

The notion of convergence in measure has played a fundamental role in the non-commutative integration theory after the elegant treatment by E. Nelson. The convergence in measure is easily expressed in terms of generalized $s$-numbers. A non-commutative version of Fatou's lemma or dominated convergence theorem is proved for sequences assumed to be

1980 Mathematics Subject Classificaion (1985 Revision) 46L50.

${ }^{*}$ Supported in part by a Grant-in-aid for Scientific Research from the Japanese Ministry of Education. 
converging in measure [FK; Lemma 3.3, Theorem 3.5]). However, for various operations of $\tau$-measurable operators, sometimes it is not enough to consider the convergence in measure. If $\tau(1)<\infty$, since the strong operator topology agrees with the $L^{2}$-norm topology induced by $\tau$ on the unit ball of $\mathscr{M}$, there exist few such problems. On the contrary, if $\tau(1)=\infty$, then even a sequence of monotone increasing projections in $\mathscr{M}$ may not converge in measure. In order to treat with such natural objects, we will introduce the notion of convergence locally in measure, which is an interpretation of the convergence almost everywhere in the commutative context.

Since several commutative methods (such as taking the supremum function of a family of functions) seem no more available, our theorems will be stated in form as how one can employ commutative results in the non-commutative context.

The first half of Section 1 consists of some preliminaries related to commutative Banach function spaces or to measurable operators. In the rest, we give a slight modification of the axioms for non-commutative Banach function spaces, and we show that the associate space itself satisfies the modified axioms.

One of the principal results of this paper (Theorem 2.5) is that if is a $\sigma$-finite semifinite factor then non-commutative Banach function spaces bijectively correspond to rearrangement invariant function norms on $(0, \tau(1))$. By the way, using some techniques developed in [H], we obtain an extension of Fatou's lemma for generalized $s$-numbers of measurable operators which seems to be important itself.

Finally, in Section 3, we show that a non-commutative Banach function space determined by a rearrangement invariant function norm has absolutely continuous norm if and only if the function norm is absolutely continuous (Theorem 3.4). As a consequence, we obtain an extension of separability condition for non-commutative Banach function spaces to general semifinite cases (Theorem 3.8).

\section{Preliminaries}

Let $(X, \mu)$ be a totally $\sigma$-finite measure space. Let $\mathfrak{M}$ be the collection of all extended complex valued $\mu$-measurable functions on $X$ and let $\mathfrak{M}_{0}$ be the class of functions in $\mathfrak{M}$ that are finite $\mu$-a.e. We denote by $L_{\rho}$ a Banach function space determined by a Banach function norm $\rho$ (see [BS ; Chapter 1, Definition 1.1] for definition). We also denote by $f^{*}$ the decreasing rearragement of $f$. Two functions $f, g \in \mathbb{M}_{0}$ are said to be equimeasurable if $f^{*}(t)=g^{*}(t), t>0$. A function norm $\rho$ is said to be $r e$ - 
arrangement invariant if $\rho(f)=\rho(g)$ for every pair of equimeasurable functions $f, g \in \mathfrak{M}_{0}$. When $f, g \in \mathfrak{M}_{0}$, we write $g<f$ if $\int_{0}^{t} g^{*}(s) d s \leq \int_{0}^{t} f^{*}(s) d s, t$ $>0$. For basic properties of rearrangement invariant Banach function spaces, the reader is referred to [BS], [CR], [L].

Next, we shall collect some fundamental definitions concerning to measurable operators. Throughout this paper, we denote by $\mathscr{M}$ a $\sigma$-finite semifinite von Neumann algebra on a Hilbert space $\mathscr{H}$ with faithful normal semifinite trace $\tau$. A closed densely defined operator $x$ on $\mathscr{H}$ with its domain $\mathscr{D}(x)$ is said to be affiliated with $\mathscr{M}$ if, for each unitary $u$ in the commutant of $\mathscr{M}$, the identity $u^{*} x u=x$ holds. Then $x$ is said to be $\tau$-measurable if, for every $\varepsilon>0$, there exists a projection $e \in \mathscr{M}$ such that $e \mathscr{H} \subset \mathscr{D}(x)$ and $\tau\left(e^{\perp}\right) \leq \varepsilon$, where $e^{\perp}$ denotes $1-e$. The set of all $\tau$-measurable operators will be denoted by $\widetilde{\mathscr{M}}$, which is a $*$-algebra with sum and product being the respective closure of the algebraic operations. For any $\varepsilon, \delta>0$, we put

$$
\begin{gathered}
N(\varepsilon, \delta)=\{x \in \widetilde{M} ; \text { there exists a projection } e \in \mathscr{M} \text { such that } \\
\left.\qquad x e \| \leq \varepsilon \text { and } \tau\left(e^{\perp}\right) \leq \delta\right\} .
\end{gathered}
$$

The family $\{N(\varepsilon, \delta) ; \varepsilon, \delta>0\}$ forms a base at 0 for a complete Hausdorff topology on $\widetilde{\mathscr{M}}$, which is called the measure topology. The proofs of these facts may be found in [N] and [Te].

Now we recall several preliminary facts related to generalized $s$-numbers of $\tau$-measurable operators. We state them here in a form suitable for our purposes. Details and proofs are found in [FK] or [H].

For a closed densely defined operator $x$ affiliated with $\mathscr{M}$, the generalized $s$-number $\mu .(x)$ is defined by

$$
\mu_{t}(x)=\inf \left\{\|x e\|: e \text { is a projection in } \mathscr{M} \text { with } \tau\left(e^{\perp}\right) \leq t\right\} .
$$

Then $x$ is $\tau$-measurable if and only if $\mu_{t}(x)<\infty$ for all $t>0$. For each $x$ $\in \widetilde{\mathscr{M}}$, the map $t \rightarrow \mu_{t}(x)$ from $(0, \infty)$ into $[0, \infty)$ is non-increasing and right-continuous. If we put $\nu_{s}(x)=\tau\left(E_{(s, \infty)}(|x|)\right), s \geq 0$, then $\mu_{t}(x)=\inf \{s>0$; $\left.\nu_{s}(x) \leq t\right\}, t>0$, where $E_{(s, \infty)}(|x|)$ denotes the spectral projection of $|x|$ corresponding to the interval $(s, \infty)$.

For each $x \in \tilde{\mathscr{M}}$, the following conditions are equivalent ;

(1) $x \in \mathscr{M}+L^{1}(\mathscr{M}, \tau)$,

(2) $\int_{0}^{s} \mu_{t}(x) d t<\infty$ for some $s>0$,

(3) $|x| E_{(r, \infty)}(|x|) \in L^{1}(\mathscr{M}, \tau)$ for some $r>0$. 
An operator $x \in \widetilde{\mathscr{M}}$ is said to be $\tau$-compact if $x$ belongs to the closure of $\{x \in \mathscr{M} ; \tau(s(|x|))<\infty\}$ under the measure topology, where $s(|x|)$ denotes the support projection of $|x|$.

For each $x \in \widetilde{\mathscr{M}}$, the following conditions are equivalent;

(1) $x$ is $\tau$-compact,

(2) $\nu_{s}(x)<\infty$ for all $s>0$,

(3) $\lim _{t \rightarrow \infty} \mu_{t}(x)=0$,

(4) $|x| E_{(1 / n, n]}(|x|) \in L^{1}(\mathscr{M}, \tau)$ for all $n \geq 1$.

Now we introduce the notion of convergence locally in measure.

DEFINITION 1.1. We say that a sequence $\left\{x_{n}\right\}_{n=1}^{\infty}$ in $\widetilde{\mathscr{M}}$ converges locally in measure to an element $x$ in $\widetilde{\mathscr{M}}$ if, for any projection $p$ in $\mathscr{M}$ with $\tau(p)<\infty$, the sequence $\left\{p x_{n} p\right\}_{n=1}^{\infty}$ converges in measure to $p x p$.

REMARK 1.2. Clearly, the convergence in measure leads to the convergence locally in measure. If $\tau(1)<\infty$, then the above two convergences are equivalent each other.

Consider the case $\mathscr{M}=B(\mathscr{H})$ and $\tau=$ canonical trace. Then there exists a sequence $\left\{p_{n}\right\}_{n=1}^{\infty}$ of increasing projections such that $p_{n} \uparrow 1$ strongly and $\tau\left(p_{n}\right)=n$. It is easy to see that $\left\{p_{n}\right\}_{n=1}^{\infty}$ does not converge in measure. However, $p_{n} \uparrow 1$ locally in measure by [W1; Lemma 2.1].

In order to complete the theory of non-commutative Banach function spaces for infinite von Neumann algebras, we should modify the system of axioms for symmetric norms. We would like to take the modification as slightly as possible.

DEFINITION 1.3. A mapping $\|\cdot\| \cdot \|: \widetilde{\mathscr{M}}+\rightarrow[0, \infty]$ is called a symmetric Banach function norm (or simply symmetric norm) if, for all $x, y, x_{n} \in \widetilde{\mathscr{M}}_{+}$, for all $a \in \mathscr{M}$, for all constants $\alpha \geq 0$, and for all projections $p$ in $\mathscr{M}$, the following properties hold:

$$
\begin{aligned}
& \text { (P-I ) } \quad\|x\|\|=0 \Leftrightarrow x=0, \quad\| \alpha x\|=\alpha|\|x|\|, \quad\| x+y\||\|\leq\| x|\|+\|y \mid\| \\
& \text { (P-II) } 0 \leq y \leq x \Rightarrow\|\| y\|\mid \leq\| x\|\| \\
& \left.(\mathrm{P}-\mathrm{III})^{\prime} \quad x_{n} \rightarrow x \text { (locally in measure }\right) \Rightarrow|\|x\|| \leq \liminf \left|\left\|x_{n}\right\|\right| \\
& \text { (P-IV) } \tau(p)<\infty \Rightarrow\|p\| \mid \|<\text {, } \\
& (\mathrm{P}-\mathrm{V}) \quad \tau(p)<\infty \Rightarrow \tau(p x) \leq C_{p}\|x\| \| \\
& (\mathrm{P}-\mathrm{VI}) \quad\|\| a^{*} x a\|\| \leq\|a\|\|\| x\|\|\|a\| \text {. }
\end{aligned}
$$

For the convenience in after, we consider two conditions for a symmetric norm. 
( $h-1) \quad$ The value $\|\not|\||$ in (P-IV) depends only on the value $\tau(p)$.

$(q-2) \quad$ The constant $C_{p}$ in $(\mathrm{P}-\mathrm{V})$ depends only on the value $\tau(p)$.

Let $\||\cdot| \mid$ be a symmetric norm. We denote by $\mathbb{S}_{\|\cdot \mid\|}$ the collection of all operators $x$ in $\widetilde{\mathscr{M}}$ for which $\||| x||\|<\infty$. Define $\||x|\|$ for $x \in \mathbb{S}_{\|\| \cdot \|}$ by $\||| x||=|\|| x||||$. The statements corresponding to [W2; Lemma 1.2, Proposition 1.3] hold without any change. We summarize them as follows.

PROPOSITION 1. 4.

(1) If $x_{n}, x$ in $\varsigma_{\|\cdot \mid\|}$ and $\mid\left\|x_{n}-x\right\| \| \rightarrow 0$, then $\left|x_{n}-x\right| \rightarrow 0$ locally in measure.

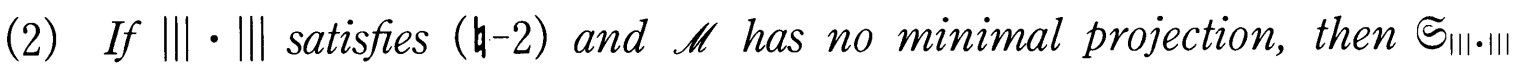
is an $\mathscr{M}$-bimodule Banach space with $\mathfrak{S}_{\|\cdot\| \|} \subset \mathscr{M}+L^{1}(\mathscr{M}, \tau)$.

Let $\||\cdot| \mid$ be a symmetric norm on $\widetilde{\mathscr{M}}$. Its associate norm \|\|$^{\prime} \|\left.\right|^{\prime}$ is defined without any change. That is,

$$
\|y \mid\|^{\prime}=\sup \left\{\tau(x \quad y): x \in \widetilde{\mathscr{M}}_{+},\|x \mid\| \leq 1\right\}, \quad y \in \widetilde{\mathscr{M}}_{+} .
$$

Proposition 1.5. Let $\||\cdot|\|$ be a symmetric norm. Then the associate norm $\|\cdot \mid\|^{\prime}$ satisfies (P-III)', and it is a symmetric norm.

ProOF. To show that $\|\cdot\|^{\prime}$ satisfies (P-III)', let $y_{n}, y \in \widetilde{\mathscr{M}}_{+}$and $y_{n}$ $\rightarrow y$ locally in measure. Put $e_{k}=E_{[0, k]}(y)$ and $y^{(k)}=y e_{k}$. Fix a number $k$. Take a family $\left\{p_{j}\right\}_{j=1}^{\infty}$ of increasing projections in $\mathscr{M}$ such that $\tau\left(p_{j}\right)<\infty$ and $p_{j} \uparrow e_{k}$ strongly. Then we have

$$
p_{j} y_{n} p_{j} \rightarrow p_{j} y p_{j}=p_{j} y^{(k)} p_{j} \text { in measure as } n \rightarrow \infty .
$$

Hence, for any element $x$ in $\mathscr{M}+$ with $\|x\| \leq 1$,

$$
x^{1 / 2} p_{j} y_{n} p_{j} x^{1 / 2} \rightarrow x^{1 / 2} p_{j} y^{(k)} p_{j} x^{1 / 2} \text { in measure as } n \rightarrow \infty .
$$

It follows from the Fatou's lemma of trace version that

$$
\begin{aligned}
\tau\left(x^{1 / 2} p_{j} y^{(k)} p_{j} x^{1 / 2}\right) & \leq \liminf _{n \rightarrow \infty} \tau\left(x^{1 / 2} p_{j} y_{n} p_{j} x^{1 / 2}\right) \\
& =\liminf _{n \rightarrow \infty} \tau\left(y_{n}^{1 / 2} p_{j} x p_{j} y_{n}^{1 / 2}\right) .
\end{aligned}
$$

Since $\left\|p_{j} x p_{j}\right\| \leq 1$, this implies that $\tau\left(x^{1 / 2} p_{j} y^{(k)} p_{j} x^{1 / 2}\right) \leq \liminf _{n \rightarrow \infty} \mid\left\|y_{n}\right\| \|^{\prime}$.

On the other hand, $x^{1 / 2} p_{j} y^{(k)} p_{j} x^{1 / 2} \rightarrow x^{1 / 2} y^{(k)} x^{1 / 2}$ strongly in $\mathscr{M}$ as $j \rightarrow \infty$. By the lower semi-continuity of $\tau$, we have $\tau\left(x^{1 / 2} y^{(k)} x^{1 / 2}\right) \leq \liminf _{n \rightarrow \infty}\left\|y_{n}\right\| \|^{\prime}$. Since $x^{1 / 2} y^{(k)} x^{1 / 2} \uparrow x^{1 / 2} y x^{1 / 2}$ in measure, we have $\tau\left(x^{1 / 2} y^{(k)} x^{1 / 2}\right) \uparrow \tau\left(x^{1 / 2} y x^{1 / 2}\right)$. Therefore we have $\tau\left(x^{1 / 2} y x^{1 / 2}\right) \leq \liminf _{n \rightarrow \infty}\left\|y_{n}\right\|^{\prime}$. It is easy to see that 


$$
\|\mid y\|^{\prime}=\sup \left\{\tau(x y) ; x \in \mathscr{M}_{+},|\|x \mid\| \leq 1\}, \quad y \in \widetilde{\mathscr{M}}_{+} .\right.
$$

Thus the desired inequality is proved.

The normed space $\mathfrak{S}_{\|\cdot\| \|^{\prime}}$ is called the associate space of $\mathfrak{S}=\mathfrak{S}_{\|\cdot\|\|\|}$ and is simply denoted by $\varsigma^{\prime}$. We can obtain elementary facts on $\mathbb{S}^{\prime}$ such as Hölder's inequality in the same way as [W2 ; Remark 2.3-Lemma 2.6]. Here we summarize them.

PROPOSITION 1.6.

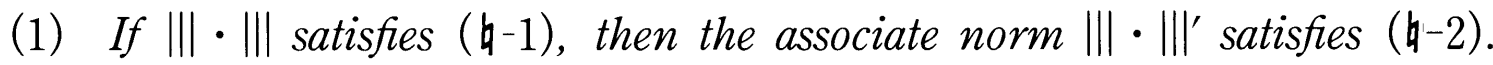

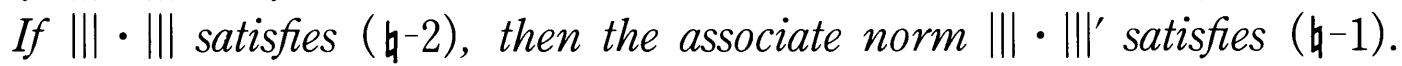

(2) Let $\mathfrak{S}^{\prime}$ be the associate space of $\mathbb{S}$. If $x \in \mathfrak{S}$ and $y \in \mathbb{S}^{\prime}$, then $x y \in L^{1}(\mathscr{M}, \tau)$ and

$$
\tau(|x y|) \leq\|\| x\|||\| y \mid \|^{\prime} .
$$

(3) Suppose that $\subseteq$ is complete. Let $y$ be an element in $\tilde{\mathscr{M}}$. Then $y$ is in $\mathbb{S}^{\prime}$ is equivalent to that $x y \in L^{1}(\mathscr{M}, \tau)$ for all $x \in \subseteq$.

THEOREM 1.7. If $\tau(1)<\infty$, or if $\||\cdot|||$ satisfies $(\boldsymbol{\natural}-1)$ and $(\mathfrak{h}-2)$ and has no minimal projection, then $\subseteq=\varsigma^{\prime \prime}$.

PROOF. At first, from the assumption, the spaces $\mathfrak{S}$ and $\mathfrak{S}^{\prime}$ are both complete. Clearly \|\|$\cdot\|\|^{\prime \prime}$ is a symmetric norm. If $x \in \subseteq$, then Hölder's inequality leads that $x \in \mathcal{S}^{\prime \prime}$ and $\||x|\|^{\prime \prime} \leq|\|x\||$.

For the converse, let $p$ be a projection in $\mathscr{M}$ with $\tau(p)<\infty$. The argument in the proof of [W2; Theorem 3.7] shows that $\||x|\| \leq \mid\|x\| \|^{\prime \prime}$ whenever $x \in p \widetilde{\mathscr{M}} p$. Choose an increasing sequence $\left\{p_{n}\right\}_{n=1}^{\infty}$ of projections in $\mathscr{M}$ which converges to 1 in the strong operator topology and satisfies $\tau\left(p_{n}\right)<\infty$. For $x \in \widetilde{\mathscr{M}}$, we have $\|\left|p_{n}\right| x\left|p_{n}\right||| \leq\left.|| p_{n}|x| p_{n}||\right|^{\prime \prime} \leq|||x|||^{\prime \prime}$. Let $q$ be a projection in $\mathscr{M}$ with $\tau(q)<\infty$. Since $q p_{n} \rightarrow q$ in measure by [W1; Lemma 2.1], we have $q p_{n}|x| p_{n} q \rightarrow q|x| q$ in measure. Hence $p_{n}|x| p_{n} \rightarrow|x|$ locally in measure, so (P-III)' implies that ||$x||\left|\leq \liminf _{n \rightarrow \infty}\right|\left|p_{n}\right| x\left|p_{n} \|\right|$. Thus we get the desired inequality $\|x\||| \leq\|x \mid\|^{\prime \prime}$.

\section{Correspondence between symmetric norms and R. I. function norms}

Recall that a measure space $(X, \mu)$ is said to be resonant if, for each pair of measurable functions $f$ and $g$ with finite absolute values almost everywhere, the identity

$$
\int_{0}^{\infty} f^{*}(t) g^{*}(t) d t=\sup \int_{X}|f \widetilde{g}| d \mu
$$


holds, where the supremum is taken over all functions $\widetilde{g}$ on $X$ equimeasurable with $g$. In [H], Hiai introduced an analogue of equimeasurability for measurable operators. A pair $x, y \in \widetilde{\mathscr{M}}$ is said to be spectral equivalent if $\mu .(x)=\mu .(y)$ and denoted by $x \sim y$ (in [H], it is denoted by $x \approx y$ ).

DEFINITION 2.1. ( $\mathscr{M}, \tau)$ is said to be resonant if, for each pair of elements $x$ and $y$ in $\widetilde{\mathscr{M}}_{+}$, the identity holds;

$$
\int_{0}^{\infty} \mu_{t}(x) \mu_{t}(y) d t=\sup \left\{\tau(x \tilde{y}) ; \tilde{y} \in \tilde{\mathscr{M}}_{+}, \tilde{y} \sim y\right\} .
$$

PROPOSITION 2.2. Let $\mathscr{M}$ be a semifinite von Neumann algebra with a faithful normal semifinite trace $\tau$. If $\mathscr{M}$ has no minimal projection, then $(\mathscr{M}, \tau)$ is resonant.

To prove this proposition, we need the following lemma. It is an extension of [FK ; Lemma 3.4], and it plays a crucial role in this article.

LEMMA 2.3. Suppose that $\mathscr{M}$ has no minimal projection. Let $x \in$ $\widetilde{\mathscr{M}}_{+}$and let $\left\{x_{n}\right\}_{n=1}^{\infty}$ be a sequence in $\widetilde{\mathscr{M}}_{+}$. If $x_{n} \rightarrow x$ locally in measure, then $\mu_{t}(x) \leq \liminf _{n \rightarrow \infty} \mu_{t}\left(x_{n}\right)$ for each $t>0$.

PROOF. Let $p$ be a projection in $\mathscr{M}$ with $\tau(p)<\infty$. Since $p x_{n} p \rightarrow p x p$ in measure, we obtain by [FK ; Lemma 3.4] that $\mu_{t}(p x p) \leq \liminf _{n \rightarrow \infty} \mu_{t}\left(p x_{n} p\right)$ $\leq \liminf _{n \rightarrow \infty} \mu_{t}\left(x_{n}\right)$ for each $t>0$. Now we divide the proof into three cases.

Case (1) Suppose that $\lim _{t \rightarrow \infty} \mu_{t}(x)=0$. This is equivalent to that $\tau\left(E_{(s, \infty)}(x)\right)<\infty$ for each $s>0$. Putting $p_{k}=E_{(1 / k, \infty)}(x)$, we have $\tau\left(p_{k}\right)<\infty$ and $p_{k} x p_{k} \uparrow x$ in measure. Hence $\mu_{t}\left(p_{k} x p_{k}\right) \uparrow \mu_{t}(x)$ for each $t>0$.

Case (2) Suppose that $\lim _{t \rightarrow \infty} \mu_{t}(x)=\alpha>0$ and $\mu_{t}(x)>\alpha$ for all $t>0$. Putting $p_{k}=E_{(\alpha+1 / k, \infty)}(x)$, we have $\tau\left(p_{k}\right)<\infty$ and

$$
E_{(s, \infty)}\left(p_{k} x p_{k}\right)=\left\{\begin{array}{lll}
E_{(\alpha+1 / k, \infty)}(x) & \text { if } & 0<s<\alpha+1 / k, \\
E_{(s, \infty)}(x) & \text { if } & s \geq \alpha+1 / k .
\end{array}\right.
$$

Therefore we get

$$
\mu_{t}\left(p_{k} x p_{k}\right)=\left\{\begin{array}{lll}
\mu_{t}(x) & \text { if } \quad 0<t<\tau\left(p_{k}\right), \\
0 & \text { if } \quad t \geq \tau\left(p_{k}\right) .
\end{array}\right.
$$

Since $\tau\left(p_{k}\right) \uparrow \infty$ by $\mu_{t}(x)>\alpha$ for all $t>0$, we obtain $\mu_{t}\left(p_{k} x p_{k}\right) \uparrow \mu_{t}(x)$ for each $t>0$. 
Case (3) Suppose that $\lim _{t \rightarrow \infty} \mu_{t}(x)=\alpha>0$ and $\mu_{t}(x)=\alpha$ for some $t>0$. This is equivalent to that $\tau\left(E_{(\alpha, \infty)}(x)\right)<\infty, \tau\left(E_{(\alpha-\varepsilon, \infty)}(x)\right)=\infty, \forall \varepsilon>0$. Note that $\tau\left(E_{(\alpha-\varepsilon, \alpha]}(x)\right)=\infty, \forall \varepsilon>0$. Since $\mathscr{M}$ has no minimal projection, we can take a projection $q_{k} \leq E_{(\alpha-1 / k, \alpha]}(x)$ such that $k \leq \tau\left(q_{k}\right)<\infty$. Putting $p_{k}=$ $E_{(\alpha, \infty)}(x)+q_{k}$, we have

$$
\begin{gathered}
\tau\left(p_{k}\right)<\infty, \\
p_{k} x p_{k}=x E_{(\alpha, \infty)}(x)+q_{k} x q_{k} \quad \text { and } \\
\left(\alpha-\frac{1}{k}\right) q_{k} \leq q_{k} x q_{k} \leq \alpha q_{k} .
\end{gathered}
$$

We denote $a=x E_{(\alpha, \infty)}(x)+(\alpha-1 / k) q_{k}$ and $b=x E_{(\alpha, \infty)}(x)+\alpha q_{k}$. Then it follows that $a \leq p_{k} x p_{k} \leq b$,

$$
E_{(s, \infty)}(a)=\left\{\begin{array}{lll}
E_{(\alpha, \infty)}(x)+q_{k} & \text { if } & 0<s<\alpha-1 / k \\
E_{(\alpha, \infty)}(x) & \text { if } & \alpha-1 / k \leq s<\alpha, \\
E_{(s, \infty)}(x) & \text { if } & s \geq \alpha
\end{array}\right.
$$

and

$$
E_{(s, \infty)}(b)=\left\{\begin{array}{lll}
E_{(\alpha, \infty)}(x)+q_{k} & \text { if } \quad 0<s<\alpha \\
E_{(s, \infty)}(x) & \text { if } \quad s \geq \alpha
\end{array}\right.
$$

Put $t_{0}=\tau\left(E_{(\alpha, \infty)}(x)\right), t_{k}=\tau\left(E_{(\alpha, \infty)}(x)+q_{k}\right)$. It follows from elementary properties of $s$-numbers $\mu .(x)$ and distribution functions $\tau\left(E_{(\cdot, \infty)}(x)\right)$ that $t_{0}=\inf \left\{t>0 ; \mu_{t}(x)=\alpha\right\}$. Then we get

$$
\mu_{t}(a)=\left\{\begin{array}{lll}
\mu_{t}(x) & \text { if } & 0<t<t_{0} \\
\alpha-1 / k & \text { if } & t_{0} \leq t<t_{k} \\
0 & \text { if } & t \geq t_{k}
\end{array}\right.
$$

and

$$
\mu_{t}(b)=\left\{\begin{array}{lll}
\mu_{t}(x) & \text { if } \quad 0<t<t_{k} \\
0 & \text { if } \quad t \geq t_{k}
\end{array}\right.
$$

Hence we have $\mu_{t}\left(p_{k} x p_{k}\right)=\mu_{t}(x)$ if $0<t<t_{0}$ and $\alpha-1 / k=\mu_{t}(a) \leq \mu_{t}\left(p_{k} x p_{k}\right) \leq$ $\mu_{t}(b)=\alpha$ if $t_{0} \leq t<t_{k}$.

Fix an arbitrary number $t \geq t_{0}$. Since $k \leq \tau\left(q_{k}\right) \rightarrow \infty$, we can find a number $k_{0}$ such that $t_{0} \leq t<t_{k}$ for each $k \geq k_{0}$. Then we have $\alpha-1 / k \leq$ $\mu_{t}\left(p_{k} x p_{k}\right) \leq \alpha$ for each $k \geq k_{0}$. Hence $\mu_{t}\left(p_{k} x p_{k}\right) \rightarrow \alpha=\mu_{t}(x)$. Thus we conclude that $\mu_{t}\left(p_{k} x p_{k}\right) \rightarrow \mu_{t}(x)$ for each $t>0$.

In any case, taking the supremum in the inequality $\mu_{t}(p x p) \leq$ 
$\liminf _{n \rightarrow \infty} \mu_{t}\left(x_{n}\right), \forall t>0$ over projections $p$ in $\mathscr{M}$ with $\tau(p)<\infty$, we have the desired inquality $\mu_{t}(x) \leq \liminf _{n \rightarrow \infty} \mu_{t}\left(x_{n}\right), \forall t>0$.

ProOF OF PROPOSITION 2.2. We may assume $0<\int_{0}^{\infty} \mu_{t}(x) \mu_{t}(y) d t$. Fix any number $\beta$ satisfying $0<\beta<\int_{0}^{\infty} \mu_{t}(x) \mu_{t}(y) d t$. We can choose a sequence $\left\{p_{n}\right\}_{n=1}^{\infty}$ of projections in $\mathscr{M}$ such that $p_{n} \uparrow 1$ strongly, $\tau\left(p_{n}\right)<\infty$ and each $p_{n}$ commutes with $y$. Then $x^{1 / 2} p_{n} x^{1 / 2} \uparrow x$ and $y^{1 / 2} p_{n} y^{1 / 2} \uparrow y$ locally in measure. By the previous lemma and the monotone convergence theorem, there exists a number $N$ such that

$$
\beta<\int_{0}^{\infty} \mu_{t}\left(x^{1 / 2} p_{N} x^{1 / 2}\right) \mu_{t}\left(y^{1 / 2} p_{N} y^{1 / 2}\right) d t=\int_{0}^{\infty} \mu_{t}\left(p_{N} x p_{N}\right) \mu_{t}\left(p_{N} y p_{N}\right) d t .
$$

Now $\left(p_{N} \mathscr{M} p_{N}, \tau\left(p_{N} \cdot p_{N}\right)\right)$ is strongly resonant by [W2 ; Proposition 3. 2]. We can therefore take a positive element $z \in p_{N} \widetilde{\mathscr{M}} p_{N}$ with $z \sim p_{N} y p_{N}$ such that

$$
\int_{0}^{\tau\left(p_{N}\right)} \mu_{t}\left(p_{N} x p_{N}\right) \mu_{t}\left(p_{N} y p_{N}\right) d t=\tau\left(p_{N} x z p_{N}\right) .
$$

It follws from $\mu_{t}\left(p_{N} x p_{N}\right)=0, \forall t \geq \tau\left(p_{N}\right)$ that $\beta<\tau\left(p_{N} x z p_{N}\right)=\tau(x z)$. Put $\tilde{y}$ $=z+p_{N}^{\perp} y p_{N}^{\perp}$. Since $p_{N}$ commutes with $y$, we have

$$
\begin{aligned}
\mu_{t}(\tilde{y}) & =\inf \left\{s \geq 0 ; \tau\left(E_{(s, \infty)}(z)\right)+\tau\left(E_{(s, \infty)}\left(p_{N}^{\perp} y p_{N}^{\perp}\right)\right) \leq t\right\} \\
& =\inf \left\{s \geq 0 ; \tau\left(E_{(s, \infty)}\left(p_{N} y p_{N}\right)\right)+\tau\left(E_{(s, \infty)}\left(p_{N}^{\perp} y p_{N}^{\perp}\right)\right) \leq t\right\} \\
& =\inf \left\{s \geq 0 ; \tau\left(E_{(s, \infty)}(y)\right) \leq t\right\} \\
& =\mu_{t}(y) .
\end{aligned}
$$

Hence $\beta<\tau(x z) \leq \tau(x \tilde{y})$. This completes the proof.

The following lemma is shown by the previous proposition and Theorem 1.7 as in the proof of [W2; Lemma 3.3].

LEMMA 2.4. Let $\mathscr{M}$ be a semifinite von Neumann algebra which has no minimal projection. Let $\||\cdot| \mid$ be a symmetric norm. Suppose the following condition (\#) is satisfied;

$$
\mu .(x)=\mu .(y) \text { almost everywhere } \Rightarrow\||| x \mid\|=\|y\| \|, \quad x, y \in \tilde{\mathscr{M}} .
$$

Then the following identities hold;

$$
\||y|\|^{\prime}=\sup \left\{\int_{0}^{\infty} \mu_{t}(x) \mu_{t}(y) d t ;\|x\| \mid \leq 1\right\}, \quad y \in \widetilde{\mathscr{M}}_{+}
$$




$$
\||x|\|=\sup \left\{\int_{0}^{\infty} \mu_{t}(x) \mu_{t}(y) d t ;\|y \mid\|^{\prime} \leq 1\right\}, \quad x \in \tilde{\mathscr{M}}_{+} .
$$

Now we are in a position to characterize the symmetric norms in the case of $\mathscr{M}$ is a $\sigma$-finite semifinite factor.

THEOREM 2.5. Let $\mathscr{M}$ be a $\sigma$-finite semifinite factor and let $\tau$ be a faithful normal semifinite trace on $\mathscr{M}$. Then there exists a bijective correspondence between the collection of all symmetric norms $\|\cdot\| \|$ on $\widetilde{\mathscr{M}}$ and the collection of all rearrangement invariant function norms $\rho$ on $\mathfrak{M}_{0}((0$, $\tau(1)), m)$, where $m$ denotes the Lebesgue measure.

PROOF. We may assume that $\mathscr{M}$ is a factor of type $\mathrm{II}_{\infty}$. Suppose that $\rho$ is a rearrangement invariant function norm on $\mathfrak{M}_{0}=\mathfrak{M}_{0}((0, \infty), m)$. Define $\|x\|_{\rho}=\rho(\mu .(x))$. As in the proof of [DDP: Theorem 4.2], we already have $\left\|x+y\left|\left\|_{\rho} \leq\right\| x\right|\right\|_{\rho}+\|y\| \|_{\rho}$ by the majorization $\mu .(x+y)$ $<\mu .(x)+\mu .(y), x, y \in \widetilde{M}$. For (P-III)', suppose that $x_{n} \rightarrow x$ locally in measure as $n \rightarrow \infty$. Then we have $\mu .(x) \leq \liminf \mu .\left(x_{n}\right)$ by Lemma 2.3. Hence

$$
\|\| x\left\|_{\rho}=\rho(\mu .(x)) \leq \rho\left(\liminf \mu .\left(x_{n}\right)\right) \leq \liminf \rho\left(\mu .\left(x_{n}\right)\right)=\liminf \mid\right\| x_{n} \|_{\rho} .
$$

Conversely, let $\|\cdot \cdot\|$ be a symmetric norm on $\widetilde{\mathscr{M}}$. Considering $m$ as a trace on $L^{\infty}((0, \infty), m)$, let $\mathfrak{M}_{1}$ be the set of all functions whose multiplication operators are $m$-measurable. Note that $L^{\infty}+L^{1} \subset \mathfrak{M}_{1}$. For any $f \in$ $\mathfrak{M}_{1}$, we want to take an element $x \in \widetilde{\mathscr{M}}_{+}$such that $f^{*}=\mu .(x)$ almost everywhere, and to define as $\rho_{\|\cdot\| \|}(f)=\|\| x\|\|$. Since $\mathscr{M}$ has no minimal projection, there exists a family of increasing projections $\left\{e_{t}\right\}_{0 \leq t<\infty}$ in $\mathscr{M}$ such that $e_{t} \uparrow 1$ strongly and $\tau\left(e_{t}\right)=t$. If we put $x=\int_{0}^{\infty} f^{*}(t) d e_{t}$, then we have $\mu .(x)=f^{*}$ and $x$ is $\tau$-measurable. Suppose that $y \in \tilde{\mathscr{M}}$ is another element satisfying $\mu .(x)=\mu .(y)=f^{*}$. It follows from [H ; Proposition $\left.1.2(1)\right]$ that the condition $x \in \mathscr{M}+L^{1}(\mathscr{M}, \tau)$ is equivalent to $y \in \mathscr{M}+L^{1}(\mathscr{M}, \tau)$. If $x \notin$ $\mathscr{M}+L^{1}(\mathscr{M}, \tau)$, then we have $\|x\|\|=\infty=\| \mid\|y\|$ by Proposition 1.4. Assume that $x \in \mathscr{M}+L^{1}(\mathscr{M}, \tau)$. Take an increasing sequence $\left\{p_{n}\right\}_{n=1}^{\infty}$ of projections in $\mathscr{M}$ such that $p_{n} \uparrow 1$ strongly and $\tau\left(p_{n}\right)<\infty$. Then $\mu_{t}\left(p_{n} y p_{n}\right) \leq \mu_{t}(x)$ for all $t>0$ and each $p_{n} y p_{n}$ is a $\tau$-compact operator, that is $\mu_{t}\left(p_{n} y p_{n}\right) \rightarrow 0$ as $t \rightarrow \infty$. By the assumption that $\mathscr{M}$ is a factor and by [H; Theorem 3 . $1]$, we can conclude that $\left\|p_{n} y p_{n}\right\||\leq|\|x \mid\|$. It is clear that $p_{n} y p_{n} \rightarrow y$ locally in measure. By (P-III)', we get $\|y\|\left\|\leq \liminf \left|\left\|p_{n} y p_{n}\right\|\right| \leq\right\| \mid\|x\|$. Similarly we have $\||| x \mid\| \leq\|y\| \|$, so the condition (\#) is satisfied and the value $\rho_{\|\cdot\| \cdot \|}(f)$ is independent of the choice of an element such that $\mu .(x)=f^{*}$. Thus $\rho_{\|\| \cdot\|\|}$ is well-defined. If $f \notin \mathfrak{M}_{0}$, then we define as $\rho_{\|! \cdot\|}(f)=\infty$. We simply denote $\rho=\rho_{\|! \cdot\| \cdot}$. 
Suppose that $g<f$. Take an elements $x, y \in \widetilde{\mathscr{M}}_{+}$such that $\mu .(x)=f^{*}$, $\mu .(y)=g^{*}$, respectively. It follows from Hardy's Lemma (cf. [BS ; Proposition 3.6]) and Lemma 2.4 that $\rho(g)=\|\| y\|\leq\|\|x\|=\rho(f)$.

Let $f, g \in \mathfrak{M}_{0}$. We put $x=\int_{0}^{\infty} f^{*}(t) d e_{t}, f_{k}=f^{*} \chi_{(0, k)}$ and $x_{k}=\int_{0}^{\infty} f_{k}(t) d e_{t}$. We also define $g_{k}$ and $y_{k}$ similarly. Then we have $x-x_{k}=\int_{[k, \infty)} f^{*}(t) d e_{t}=$ $x\left(1-e_{k}\right)$. It follows that $x_{k} \uparrow x$ locally in measure. By (P-III)', we have $\rho\left(f_{k}\right)=\left|\left\|x_{k}\left|\left\|\uparrow\left|\|x \mid\|=\rho(f)\right.\right.\right.\right.\right.$. Since $(f+g)^{*} \chi_{(0, k)}<\left(f^{*}+g^{*}\right) \chi_{(0, k)}=f_{k}+g_{k}=\left(f_{k}\right.$ $\left.+g_{k}\right)^{*}$ for each $k>0$, we have

$$
\begin{aligned}
\rho(f+g) & =\sup _{k} \rho\left((f+g)^{*} \chi_{(0, k)}\right) \\
& \leq \sup _{k} \rho\left(\left(f^{*}+g^{*}\right) \chi_{(0, k)}\right) \\
& =\sup _{k}\left\|\left|x_{k}+y_{k}\right|\right\| \leq \sup _{k}\left\|x _ { k } \left|\left\|+\sup _{k}\left|\left\|y_{k}\right\|\right|\right.\right.\right. \\
& =\sup _{k} \rho\left(f_{k}\right)+\sup _{k} \rho\left(g_{k}\right)=\rho(f)+\rho(g) .
\end{aligned}
$$

For (P3) in [BS ; Chapter 1, Definition 1.1], let $0 \leq f_{n} \uparrow f$ almost everywhere. Then for any $k>0$, we have $f_{n}^{*} \chi_{(0, k)} \uparrow f^{*} \chi_{(0, k)}$ in measure. If we define $x, x_{k}$ as in the above argument and

$$
x_{n, k}=\int_{0}^{\infty} f_{n}^{*} \chi_{(0, k)}(t) d e_{t},
$$

then

$$
\begin{aligned}
& x_{n, k} \uparrow x_{k} \text { in measure as } n \uparrow \infty, \\
& x_{n, k} \uparrow x_{n} \text { locally in measure as } k \uparrow \infty \text { and } \\
& x_{k} \uparrow x \text { locally in measure as } k \uparrow \infty .
\end{aligned}
$$

It follows from (P-III) that

$$
\rho(f)=\left|\left\|x\left|\left\|=\sup _{k}|| x_{k}||=\sup _{k, n}||\left|x_{n, k}\right|\right\|=\sup _{n}\right|\right\| x_{n} \|\right|=\sup _{n} \rho\left(f_{n}\right) .
$$

(P4) follows from (P-IV).

Finally, for any measurable set $E$ and for $0 \leq f \in \mathfrak{M}_{0}$,

$$
\begin{aligned}
\int_{E} f_{k}(t) d t & \leq \int_{(0, m(E))} f_{k}^{*}(t) d t \leq \int_{(0, m(E))} \mu_{t}\left(x_{k}\right) d t \\
& =\tau\left(e_{m(E)} x_{k} e_{m(E)}\right) \leq C_{E} \mid\left\|x_{k}\right\| \leq C_{E} \rho(f),
\end{aligned}
$$

by $(\mathrm{P}-\mathrm{V})$. Letting $k \rightarrow \infty$, we have (P5).

Obviously, this correspondence in bijective. This completes the proof. 
REMARK 2.6. In the proof of the previous theorem, we saw that, if is a factor, then any symmetric norm satisfies the condition (\#).

Suppose that $\mathscr{M}$ has no minimal projection. If a symmetric norm

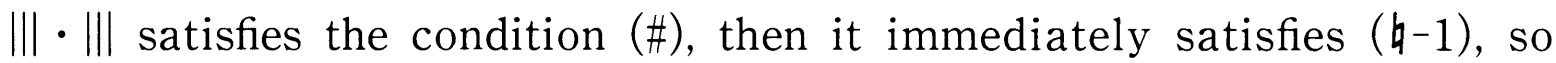
$\left\||\||^{\prime}\right.$ satisfies (h-2). Moreover, by the identity

$$
\|y \mid\|^{\prime}=\sup \left\{\int_{0}^{\infty} \mu_{t}(x) \mu_{t}(y) d t ;\|x \mid\| \leq 1\right\}, \quad y \in \widetilde{\mathscr{M}}_{+},
$$

if $\mu .(x)=\mu .(y)$ then we have $\|x \mid\|^{\prime}=\|\| y \|^{\prime}$. Therefore, $\|\left.|\cdot|\right|^{\prime}$ satisfies $(\mathfrak{h}-1)$, so $\||\cdot|||$ satisfies $(h-2)$, too.

\section{Absolutely continuity of symmetric norms}

In this section we study absolutely continuity of a symmetric norm $\||||| \mid$ which is defined by a rearrangement invariant function norm $\rho$. We also consider the separability of $\widetilde{S}_{\|\| \cdot \| \rho}$.

Recall that a function $f \in L_{\rho}$ is said to have absolutely continuous norm if $\rho\left(f \chi_{E_{n}}\right) \rightarrow 0$ for every sequence $\left\{E_{n}\right\}_{n=1}^{\infty}$ of measurable sets satisfying $\chi_{E_{n}} \rightarrow 0$ almost everywhere. A function norm $\rho$ is said to be absolutely continuous if each function in $L_{\rho}$ has absolutely continuous norm.

Let $\left\{E_{n}\right\}_{n=1}^{\infty}$ be a sequence of measurable sets satisfying $\chi_{E_{n}} \rightarrow 0$ almost everywhere. Then the sequence $F_{n}=\bigcup_{k \geq n} E_{k}(n=1,2, \cdots)$ is decreasing and $\chi_{F_{n}} \downarrow 0$. So one can see the following fact. A function $f$ has absolutely continuous norm if and only if $\rho\left(f \chi_{E_{n}}\right) \downarrow 0$ for every sequence $\left\{E_{n}\right\}_{n=1}^{\infty}$ satisfying $\chi_{E_{n}} \downarrow 0$ almost everywhere (cf. [BS ; Chapter 1, Proposition 3.2]).

Let $\left\{p_{n}\right\}_{n=1}^{\infty}$ be a sequence of projections satisfying $p_{n} \rightarrow 0$ in the strong operator topology. Although the sequence $q_{n}=\bigvee_{k \geq n} p_{k}(n=1,2, \cdots)$ is decreasing, we can not assure that $q_{n} \downarrow 0$ strongly.

EXAMPLE 3.1. Let $\left\{x_{n}\right\}_{n=1}^{\infty}$ be a sequence of unit vectors in $L^{2}([0,1])$ defined by

$$
x_{n}(t)=\sqrt{2 n+1} t^{n}, \quad t \in[0,1] .
$$

If we define one-rank projections by $p_{n}=x_{n} \otimes x_{n}$, then $p_{n} \rightarrow 0$ strongly and $q_{n}=\bigvee_{k \geq n} p_{k}=1$ for each $n$.

As a formulation considering semifinite cases in general, we should define the absolute continuity for an element in a non-commutative Banach function spaces as follows. 
Definition 3.2. Let $\|\cdot\| \|$ be a symmetric norm on $\tilde{M}$. An element $x \in \subseteq=\widetilde{S}_{\|\cdot\| \cdot \|}$ is said to have absolutely continuous norm if $\left\|x p_{n}\right\| \downarrow 0$ for every sequence $\left\{p_{n}\right\}_{n=1}^{\infty}$ of projections in $\mathscr{M}$ satisfying $p_{n} \downarrow 0$ in the strong operator topology. The set of all elements in $\subseteq$ of absolutely continuous norm is denoted by $\mathfrak{S}_{a}$. If $\subseteq \subseteq=\subseteq_{a}$, then $\||\cdot|\|$ itself is said to have absolutely continuous norm.

LEMMA 3.3. Let $\|\cdot\| \|$ be a symmetric norm on $\tilde{M}$. Let $x \in \widetilde{M}$. Let $p$ and $q$ be projections in $\mathscr{M}$ such that $q \leq p$. Then we have

(1) $\quad|| x p|\||=|||| x|p|||$

(2) $\quad\|x q\||\|\leq \mid\| x p\|\|$

PROOF. (1) is clear by the polar decomposition. So we may assume that $x \geq 0$ to show (2). Since $\mu .(x p)=\mu .(x p x)^{1 / 2}$, we have $\mu .(x q) \leq \mu .(x p)$ and $\||| x q\||=\rho(\mu .(x q)) \leq \rho(\mu .(x p))=|\|x p\| \|$.

THEOREM 3.4. Suppose that $\mathscr{M}$ is a $\sigma$-finite semifinite von Neumann algebra which has no minimal projection. Let $\rho$ be a rearrangement invariant function norm. Let $\left\|\left|\cdot\left\|=\left|\|\cdot \mid\|_{\rho}\right.\right.\right.\right.$ be the symmetric norm determined by $\rho$. An element $x \in \mathbb{S}_{\|! \cdot\|}$ has absolutely continuous norm if and only if $\mu .(x)$ has absolutely continuous norm in $L_{\rho}$. Especially, $\||\cdot|\|$ is absolutely continuous if and only if $\rho$ is absolutely continuous.

PRoof. Clearly we may assume that $x \geq 0$. From the assumption, for an arbitrary $x \in \mathbb{S}_{+}$, there exists a family $\left\{e_{t}\right\}_{0 \leq t<\tau(1)}$ of increasing projections such that $x=\int_{0}^{\tau(1)} \mu_{t}(x) d e_{t}$ and $\tau\left(e_{t}\right)=t$ (cf. [HN2; the proof of Theorem 4. 4]).

Suppose that $x$ has absolutely continuous norm. Let $\left\{E_{n}\right\}_{n=1}^{\infty}$ be a sequence of measurable subsets of $(0, \tau(1))$ such that $\chi_{E_{n}} \downarrow 0$ almost everywhere. Putting $p_{n}=\int_{0}^{\tau(1)} \chi_{E_{n}}(t) d e_{t}$, we have $p_{n} \downarrow 0$ strongly, by the dominated convergence theorem (applied to $d\left\|e_{t} \xi\right\|^{2}, \xi$ is a vector). Since $\rho$ is rearrangement invariant, we have $\rho\left(\mu,(x) \chi_{E_{n}}\right)=\rho\left(\mu .\left(x p_{n}\right)\right)=\mid\left\|x p_{n}\right\| \downarrow 0$. This implies that $\mu .(x)$ has absolutely continuous norm.

To prove the converse, suppose that $\mu(x) \in L_{\rho}$ has absolutely continuous norm. Let $\left\{p_{n}\right\}_{n=1}^{\infty}$ be an arbitrary family of projections in $\mathscr{M}$ such that $p_{n} \downarrow 0$ strongly. Put $x_{n}=\left(x p_{n} x\right)^{1 / 2}$. For each projection $q$ in $\mathscr{M}$ with $\tau(q)<\infty$, we have $q x_{n}^{2} q \rightarrow 0$ in measure and $\mu .\left(q x_{n}^{2} q\right)^{1 / 2}=\mu\left(x_{n} q x_{n}\right)^{1 / 2} \leq \mu .(x)$. It follows from [BS ; Chapter 1, Proposition 3. 6] that $\left\|q x_{n} q\right\| \| \leq$ $\rho\left(\mu .\left(q x_{n}^{2} q\right)^{1 / 2}\right) \downarrow 0$. Fix an arbitrary positive number $\varepsilon$. Since $\mu .(x)$ has absolutely continuous norm, we can take a number $k$ such that $\left\|x\left(1-e_{k}\right)\right\| \|$ 
$=\rho\left(\mu .\left(x\left(1-e_{k}\right)\right)\right)=\rho\left(\mu .(x) \chi_{(k, \infty)}\right)<\varepsilon$. Then there exists a number $n_{0}$ such that $\rho\left(\mu .\left(e_{k} x_{n}^{2} e_{k}\right)^{1 / 2}\right)<\varepsilon$ for any $n \geq n_{0}$ by the previous argument. Thus we have

$$
\| e_{k} x_{n} e_{k}^{\perp}||=\rho\left(\mu .\left(e_{k} x_{n} e_{k}^{\perp}\right)\right)=\rho\left(\mu .\left(e_{k} x_{n} e_{k}^{\perp} x_{n} e_{k}\right)^{1 / 2}\right) \leq \rho\left(\mu .\left(e_{k} x_{n}^{2} e_{k}\right)^{1 / 2}\right)<\varepsilon .
$$

Similarly, $\left\|\left|e_{k} x_{n} e_{k}\right|\right\|, \|\left|e_{k}^{\perp} x_{n} e_{k}\right| \mid<\varepsilon$. We also obtain $\left\|\left|e_{k}^{\perp} x_{n} e_{k}^{\perp}\right||\leq|\right\| e_{k}^{\perp} x e_{k}^{\perp} \mid \|$ $<\varepsilon$ for any $n \geq n_{0}$. Therefore we get

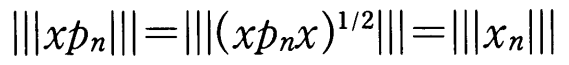

$$
\begin{aligned}
& \leq||\left|e_{k} x_{n} e_{n}\right|||+||\left|e_{k}^{\perp} x_{n} e_{k}\right| \|+||\left|e_{k} x_{n} e_{k}^{\perp}\right|||+|| e_{k}^{\perp} x_{n} e_{k}^{\perp}|| \mid \\
& <4 \varepsilon
\end{aligned}
$$

for any $n \geq n_{0}$. Thus $x$ has absolutely continuous norm. This completes the proof.

The next proposition is similarly proved as [W2; Proposition 4. 3].

Proposition 3.5. Suppose that $\mathscr{M}$ has no minimal projection. Let $x$ $\in \mathfrak{S}=\mathfrak{S}_{\|\cdot\| \cdot \|}$. Consider the following conditions :

(1) whenever $x_{n}$ and $y$ are in $\widetilde{\mathscr{M}}$ satisfying $\mu_{.}\left(x_{n}\right) \leq \mu .(x)$ and $x_{n} \rightarrow y$ locally in measure, then $\left\|x_{n}-y\right\| \| \rightarrow$,

(2) $x$ has absolutely continuous norm,

(3) whenever $x_{n}$ and $y$ are in $\widetilde{\mathscr{M}}$ satisfying $\mu .\left(x_{n}\right) \leq \mu .(x)$ and $x_{n} \rightarrow y$ in measure, then $\left\|\left|x_{n}-y\right|\right\| \rightarrow 0$.

Then we have implications $(1) \Rightarrow(2) \Rightarrow(3)$.

Let $\|\cdot\| \|$ be a symmetric norm on $\tilde{\mathscr{M}}$. In [W2; Definition 4.4], we defined $\mathfrak{S}_{\|\| \cdot\|\|}^{(0)}$ as the closure of the set $\{x \in \mathscr{M} ; \tau(s(|x|))<\infty\}$ in $\mathbb{S}_{\|\| \cdot \| !}$.

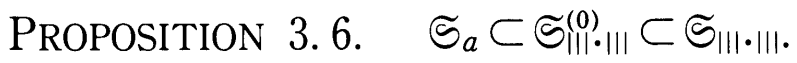

ProOF. Let $x \in \Im_{a}$. Clearly we may assume that $x \geq 0$. Let $x=$ $\int_{0}^{\infty} t d e_{t}$ be the spectral decomposition. Take a family $\left\{p_{m}\right\}_{m=1}^{\infty}$ of projections in $\mathscr{M}$ such that $p_{m} \uparrow 1$ strongly and $\tau\left(p_{m}\right)<\infty$. Put

$$
x_{n}^{(m)}=p_{m}\left(\int_{0}^{n} t d e_{t}\right) p_{m} \quad \text { and } \quad x^{(m)}=p_{m} x p_{m} .
$$

It is obvious that $x_{n}^{(m)} \in \mathscr{M}_{+}, \tau\left(s\left(x_{n}^{(m)}\right)\right) \leq \tau\left(p_{m}\right)<\infty$ and $x_{n}^{(m)} \uparrow x^{(m)}$ in measure. By Proposition 3.5, we have $\left\|x_{n}^{(m)}-x^{(m)}\right\| \downarrow 0$. Hence $x^{(m)} \in \mathbb{S}_{\|\| \cdot\|\cdot\|}^{(0)}$. Moreover, since $1-p_{m} \downarrow 0$ strongly and $x \in \Im_{a}$, we have $\left\|x-x^{(m)}\right\| \| \leq$ $\left\|x\left(1-p_{m}\right)|\|+\||\left(1-p_{m}\right) x\right\| \| \downarrow 0$. This implies that $x \in \mathbb{S}_{\|\| \cdot(0)}^{(0)} \cdot \| \cdot$ 
The second inclusion is trivial from the definition of $\mathbb{S}_{\|\| !}^{(0)} \cdot \|$.

COROLLARY 3.7. If $\rho$ is absolutely continuous, then $\mathbb{S}_{\|! \cdot\| \cdot \|}^{(0)}=\mathfrak{S}_{\|\| \cdot \| \cdot}$.

Finally, we obtain a necessary and sufficient condition for $\mathbb{S}_{\|\cdot\| \cdot \|_{\rho}}$ to be separable, in terms of $\mathscr{M}$ and $\rho$.

THEOREM 3.8. Suppose that $\mathscr{M}$ is a $\sigma$-finite semifinite von Neumann algebra which has no minimal projection. Let $\rho$ be a rearrangement invariant function norm on $\mathfrak{M}_{0}((0, \tau(1)), m)$ and let $\||\cdot|\|=|\| \cdot|||_{\rho}$. Then the symmetrically normed $\mathscr{M}$-bimodule Banach space $\mathbb{S}_{\|\cdot \cdot\|}$ is separable if and only if $\rho$ is absolutely continuous and $\mathscr{M}_{*}$ is separable.

ProOF. Suppose first that $\mathfrak{S}^{=} \mathfrak{S}_{\|\cdot\| \|}$ is separable. Take a family $\left\{e_{t}\right\}_{0 \leq t<\infty}$ of increasing projections in $\mathscr{M}$ such that $e_{t} \uparrow 1$ strongly and $\tau\left(e_{t}\right)$ $=t$. For each $f \in L_{\rho}$, we put $\Phi(f)=\int_{0}^{\infty} f(t) d e_{t} \in \widetilde{\mathscr{M}}$. Then we have $\mu .(\Phi$ $(f))=f^{*}$ and \|\|$\Phi(f) \|=\rho\left(f^{*}\right)=\rho(f)$. Thus $\Phi: L_{\rho} \rightarrow \mathbb{S}_{\|\cdot\|\|\|}$ is a linear isometric embedding. Hence $L_{\rho}$ is separable. It follows from [BS ; Chapter 1, Theorem 5.5] that $\rho$ is absolutely continuous.

Take a sequence $\left\{p_{j}\right\}_{j=1}^{\infty}$ such that $p_{j} \uparrow 1$ strongly and $\tau\left(p_{j}\right)<\infty$. Then $p_{j} \widetilde{S}_{\|! \cdot\|} p_{j}$ is a non-commutative Banach function space associated with $\left(p_{j}\right.$ $\left.\mathscr{M} p_{j}, \tau\left(p_{j} \cdot p_{j}\right)\right)$, constructed by the function norm $\left.\rho\right|_{\left(0, \tau\left(p_{j}\right)\right)}$ and it is separable. By the result of finite cases ([W2; Theorem 4.6]), we conclude that $p_{j} L^{1}(\mathscr{M}, \tau) p_{j}$ is separable. Since $\bigcup_{j=1}^{\infty} p_{j} L^{1}(\mathscr{M}, \tau) p_{j}$ is dense in $L^{1}(\mathscr{M}, \tau)$, we have the separability of $L^{1}(\mathscr{M}, \tau) \cong \mathscr{M}$ *.

Conversely, suppose that $\rho$ is absolutely continuous and $\mathscr{M}_{*}$ is separable. Notice that $\mathfrak{S}_{\|\| \cdot\|\|}^{(0)}=\mathfrak{S}_{\|\| \cdot \|}$ by Corollary 3.7. Take $\left\{p_{j}\right\}_{j=1}^{\infty}$ as above. Then we conclude that $p_{j} \Im_{\|\cdot\|} p_{j}$ is separable by the result of finite cases. Since each $x \in \mathbb{S}_{\|\cdot \cdot\|}$ has absolutely continuous norm by Theorem 3.4, we have $\left\|p_{j} x p_{j}-x\right\| \| \rightarrow$. This implies that $\bigcup_{j=1}^{\infty} p_{j} \Xi_{\|\cdot\| \|} p_{j}$ is dense in $\widetilde{S}_{\|\cdot\| \cdot \| \cdot}$ Thus $\subseteq_{\|\cdot\| ! \mid}$ is separable.

Acknowledgements. The author wishes to express his hearty thanks to Professor F. Hiai for some suggestions. This work was completed during the author's stay from May 1991 to February 1992 at Division of Applied Mathematics, Research Institute of Applied Electricity, Hokkaido University. The author is grateful to the members of Institute for their warm hospitality and support. 


\section{References}

[BS] Bennett, C. and Sharpley, R., Interpolation of Operators, Academic Press, Inc., Orland, Florida, 1988.

[CR] CHONG, K.M. and RICE, N.M., Equimeasurable rearrangements of functions, Queen's Papers in Pure and Applied Mathematics, no. 28, Queen's University, Kingston (1971).

[DDP] Doods, B.G., DOODS, T.K.-Y. and de PAGTER, B., Non-commutative Banach function spaces, Math. Z. 201 (1989), 583-597.

[FK] FACK, T. and KOSAKI, H., Generalized $s$-numbers of $\tau$-measurable operators, Pacific J. Math. 123 (1986), 269-300.

[GK] GOHBERG, I.C. and KREIN, M.G., Introduction to the theory of linear non-selfadjoint operators, Translations of Mathematical Monographs, vol. 18, Amer. Math. Soc., Rhode Island, 1969.

[H] HIAI, F., Majorization and stochastic maps in von Neumann algebras, J. Math. Anal. Appl. 127 (1987), 18-48.

[HN1] HIAI, F. and NAKAMURA, Y., Majorizations for generalized $s$-numbers in semifinite von Neumann algegras, Math. Z. 195 (1987), 17-27.

[HN2] - Distance between unitary orbits in von Neumann algebras, Pacific J. Math. 138 (1989), 259-294.

[K] KOSAKI, H., Non-commutative Lorentz spaces associated with a semifinite von Neumann algebra and applications, Proc. Japan Acad. Ser. A 57 (1981), 303 -306 .

[L] LUXEMBURG, W.A.J., Rearragement invariant Banach function spaces, Queen's Papers in Pure and Applied Mathematics, no. 10, Queen's University, Kingston (1967), 83-144.

[LS] LORENTZ, G.G. and SHIMOGAKI, T., Interpolation theorems for operators in function spaces, J. Funct. Anal. 2 (1968), 31-51.

[N] NELSON, E., Notes on non-commutative integration, J. Funct. Anal. 15 (1974), 103 -116 .

[O] OvchinniKov, V.I., s-number of measurable operators, Funct. Anal. Appl. 4 (1970), 236-242.

[Ta] TAKESAKI, M., Theory of Operator Algebras I, Springer-Verlag, New York, 1979.

[Te] TERP, M., $L^{p}$-spaces associated with von Neumann algebras, Notes, Copenhagen University (1981).

[Ti] TIKHONOV, O.E., Continuity of operator functions in topologies connected with a trace on a von Neumann algebra (Russian), Izv. Vyssh. Uchebn. Zaved. Mat., 1987, no. 1, 77-79 ; translated in Soviet Math. (Iz. VUZ), 31(1987), 110-114.

[W1] WatanABE, K., Finiteness of von Neumann algebras and non-commutative $L^{p}$-spaces, Hokkaido Math. J. 19 (1990), 297-305.

[W2] - Some results on non-commutative Banach function spaces, Math. Z. 210 (1992), 555-572.

[Y1] Yeadon, E.J., Non-commutative $L^{p}$-spaces, Math. Proc. Camb. Philos. Soc. 77 (1975), 91-102.

[Y2] - Ergodic theorems for semifinite von Neumann algebras II, Math. Proc. Camb. Philos. Soc. 88 (1980), 135-147. 